\title{
Pharmacogénétique, étude du génome et développement des médicaments
}

\author{
François Alhenc-Gelas, ${ }^{1}$ Laurent Parmentier, ${ }^{2}$ Anne Bisagni ${ }^{3}$ et les participants à la table ronde \\ $n^{\circ} 7$ de Giens XVIII ${ }^{\dagger *}$ \\ 1 INSERM, Unité 367, Paris, France \\ 2 Laboratoire GlaxoSmithKline, Marly-le-Roi, France \\ 3 INSERM, DAPS, Paris, France
}

\section{Résumé}

\begin{abstract}
Les sujets discutés concernent l'étude du polymorphisme du génome et l'identification de nouvelles cibles thérapeutiques, la place de la génétique dans le développement préclinique et clinique des médicaments, et les aspects culturels, réglementaires et logistiques du développement de la pharmacogénétique en France. Les conclusions sont que l'étude du polymorphisme génétique humain, au plan physiologique, biochimique ou génomique, a un intérêt potentiel évident pour le développement des médicaments car elle peut aider à identifier de nouvelles cibles thérapeutiques, et à mieux prévoir l'efficacité et la tolérance des médicaments. Cette dernière approche, qui repose sur l'étude de la variabilité génétique des enzymes impliquées dans le métabolisme des médicaments, et de celle des effecteurs moléculaires de l'action pharmacologique, est déjà illustrée par plusieurs exemples. La pharmacogénétique pourrait permettre, à terme, l'individualisation des traitements médicamenteux, grâce à la simplification des méthodes d'analyse des gènes et à la diminution de leur coût. Pour aider à approcher ce but encore lointain, certaines recommandations sont faites.
\end{abstract}

Mots clés : pharmacogénétique, génome, médicaments

Texte reçu le 16 juin 2003 ; accepté le 16 juin 2003

\section{Introduction}

Le développement des méthodes d'étude du génome a généré une masse importante d'informations « anatomiques » concernant les séquences des gènes et les variations interindividuelles de ces séquences. D'autres études, partant de phénotypes physiologiques simples ou plus complexes, comme la susceptibilité à développer des maladies, ont montré l'existence d'influences héréditaires sur ces phénotypes. Les deux approches sont complémentaires et leur association a permis dans un certain nombre de cas d'identifier les bases moléculaires du polymorphisme de ces phénotypes et d'établir les conséquences physiologiques de variations interindividuelles dans la séquence du génome. Ce domaine concerne a priori le développement des médicaments et ce, à plusieurs stades de ce développement. Il constitue en effet, en contribuant à identifier des gènes patho- gènes et d'autres qui jouent un rôle protecteur contre le développement des maladies, une approche possible à l'identification de nouvelles cibles médicamenteuses. Par ailleurs les enzymes impliquées dans le métabolisme des médicaments, et les molécules qui constituent les cibles pharmacologiques, peuvent présenter des variations génétiques. L'étude de ces variations peut aider à prévoir, à l'échelon de la population ou de l'individu, l'efficacité et la tolérance des médicaments. Ces concepts sont importants et ce domaine de recherche évolue rapidement. Son intérêt pour le développement des médicaments a été analysé au cours des sessions de cette table ronde.

\section{Etude du polymorphisme du génome et identification de nouvelles cibles thérapeutiques}

Une stratégie de recherche de cibles thérapeutiques par

+ Pour la liste des participants, voir en fin d'article.

* Les articles, analyses et propositions issus des Rencontres de Pharmacologie Clinique de Giens n'engagent que leurs auteurs et ne préjugent pas de la position de leur organisme de tutelle. 
l'étude du polymorphisme génétique humain et de son association avec les maladies se développe à l'heure actuelle avec des moyens importants dans plusieurs groupes pharmaceutiques, en général en collaboration avec des chercheurs académiques et des cliniciens. Elle repose sur les concepts biologiques qui aident à l'identification des bases moléculaires du polymorphisme génétique humain. Les données actuelles montrent qu'à côté du modèle de base qui associe un allèle à un caractère phénotypique, il est important de considérer des modèles plus complexes mais plus puissants d'hérédité multigénique, sous-tendus par la complexité des phénomènes d'hétérogénéité d'allèles et de locus, haplotypes et interactions entre gènes, dans la définition des phénotypes physiologiques, et le développement des maladies.

Cette approche repose sur l'étude de l'ADN de groupes de patients ou de familles atteintes, pour identifier les gènes impliqués dans le développement de la maladie, et à partir d'eux des cibles thérapeutiques potentielles. Elle peut être particulièrement intéressante dans les maladies où il n'existe pas de bons modèles de pathologie expérimentale ou d'hypothèses pathogéniques fortes, comme les maladies neuropsychiatriques.

Cette stratégie repose sur des bases théoriques solides, mais il convient de souligner certaines difficultés rencontrées dans son développement, et l'impossibilité de son évaluation, au terme actuel, au plan industriel. Au plan des difficultés pratiques, il faut mentionner le problème de l'accès aux patients pour les industriels et celui, fondamental, de la qualité du phénotypage des cohortes qui détermine in fine la validité et l'intérêt des résultats. Cette stratégie d'identification de cibles fondée sur des études d'association génétique ne peut aboutir à la mise au point d'un médicament qu'à long terme. Cela est dû à la nécessité d'obtenir un niveau de preuve suffisant par la réplication des études, d'intégrer des données complémentaires issues d'approches différentes (modèles animaux, étude du transcriptome), et de dégager les possibilités d'intervention pharmacologique à partir des gènes pathogènes ou protecteurs identifiés.

Les études d'association génétique, qu'elles soient menées dans les laboratoires académiques ou dans les laboratoires industriels, ont en outre un intérêt cognitif important, ce qui pose d'ailleurs le problème de la diffusion de leurs résultats par les industriels.

En conclusion, il apparaît important que les industriels continuent à favoriser ces stratégies à long terme, prometteuses, mais dont l'évaluation, en termes de découverte de nouveaux médicaments, ne pourra se faire avant plusieurs années.

\section{Pharmacogénétique et développement préclinique et clinique des médicaments}

L'importance des facteurs génétiques dans la variabilité interindividuelle de la réponse aux médicaments, et dans le risque de toxicité, est bien démontrée par plusieurs exemples, qui concernent en particulier le métabolisme des médicaments par les cytochromes P450, la toxicité de l'azathioprine et de l'abacavir. ${ }^{[1-3]}$

Le développement des études de pharmacogénétique, et l'évaluation de l'intérêt des analyses de génétique moléculaire pour la prescription médicamenteuse, en particulier dans les maladies fréquentes, comme les maladies cardiovasculaires et psychiatriques, le diabète et le cancer, est très souhaitable. ${ }^{[3-7]} \mathrm{Ce}$ développement peut se heurter à plusieurs obstacles :

- Conceptuel, où à l'attitude des chercheurs et des médecins qui cherchent à individualiser a priori les traitements en fonction d'hypothèses scientifiques, peut s'opposer celle des industriels et des organismes de financement des soins qui préfèrent la simplification apparente de la prescription directe sans augmentation du nombre d'analyses biologiques. Les laboratoires pharmaceutiques ont toutefois réalisé des efforts importants dans ce domaine, et pris des orientations stratégiques majeures dans le sens du développement de la pharmacogénétique au cours des phases d'études cliniques des molécules.

- Scientifique, où le constat doit être fait d'une insuffisance des connaissances sur les facteurs impliqués dans le métabolisme des médicaments, et leur variabilité génétique, ainsi que sur la variabilité génétique des cibles moléculaires des médicaments. En ce qui concerne les variations génétiques déjà identifiées ou celles qui le seront dans le futur, un point important est celui de la nécessité de l'évaluation de l'intérêt de leur étude au cours d'essais cliniques d'intervention conçus pour répondre à cette question, et pas seulement au cours des études de pharmacocinétique et de tolérance. Il serait par exemple souhaitable d'évaluer par un essai clinique l'intérêt de la détermination de certains polymorphismes des cytochromes P450 pour la prescription des antivitamines $\mathrm{K}^{[8]}$ ou celui du polymorphisme génétique des taux de l'enzyme de conversion de l'angiotensine pour la prévention cardiovasculaire et rénale par les inhibiteurs de cette enzyme. ${ }^{[9,10]}$ Par ailleurs, un nouveau domaine de la pharmacogénétique se développe, celui de l'étude du transcriptome cellulaire, pour analyser et peut-être individualiser les réponses thérapeutiques à l'échelon moléculaire.

Les recommandations issues de ces discussions sont reprises à la fin de cet article. 


\section{Aspects culturels, réglementaires et logistiques du développement de la pharmacogénétique en France}

La recherche en génétique humaine en France est encadrée par les dispositions législatives et réglementaires qui régissent la recherche clinique, les prélèvements et le stockage de l'ADN, et la constitution de fichiers informatiques. Ces dispositions, qui visent à assurer le respect de l'éthique et la protection des personnes, peuvent, dans certains cas, être perçues comme une limitation au développement des études de génétique et de pharmacogénétique en France. De nouveaux textes de loi sont attendus prochainement dans ce domaine. Il s'agit en fait des règles de rédaction du consentement éclairé et de la note d'information aux patients pour le prélèvement d'ADN, en ce qui concerne la définition de l'étendue des analyses possibles sur ces prélèvements. L'interprétation que fait le Comité Consultatif de Protection des Personnes dans la Recherche Biomédicale (CCPPRB) de ces dispositions est en général restrictive, ce qui ne permet pas l'exploitation des échantillons d'ADN au-delà des études initialement prévues, alors que l'évolution de la science et l'intérêt de la recherche biomédicale peuvent justifier de nouvelles investigations. Le cadre actuel de la loi du 20 décembre 1988, dite loi Huriet-Sérusclat, ${ }^{[11]}$ n'apparaît pas à cet égard totalement adapté aux études génétiques. Les industriels soulignent aussi la relation étroite qui existe entre la réalisation des études de pharmacogénétique et les essais cliniques princeps, prospectifs et internationaux, qui sont réalisés le plus souvent avec de fortes contraintes de temps. Les délais pour obtenir les différentes autorisations sont longs et difficilement compatibles avec la dynamique des essais prospectifs, ce qui fait craindre que ce type d'essais cliniques, comportant des études génétiques, soit mené préférentiellement dans d'autres pays européens.

Sur le plan logistique, la création prochaine des centres de ressources biologiques permettant la conservation des collections d'ADN va aider le développement de la recherche en pharmacogénétique. Certains industriels souhaitent cependant pouvoir, pour des raisons pratiques, continuer à gérer eux-mêmes leurs collections, dans le respect des règles d'éthique et des législations françaises et internationales.

\section{Conclusions et recommandations}

L'étude du polymorphisme génétique humain a un intérêt potentiel évident pour le développement des médicaments, que ce soit pour identifier de nouvelles cibles thérapeutiques ou prévoir l'efficacité et la tolérance des médicaments. La pharmacogénétique a le potentiel de permettre, à terme, l'individualisation des traitements médicamenteux, y compris au cours des maladies les plus fréquentes ${ }^{[12-15]}$ grâce en particulier au développement et à la simplification des méthodes d'analyse du polymorphisme des gènes, et à la diminution de leur coût.

Pour aider à approcher ce but encore lointain, certaines recommandations peuvent être faites :

- Chercher à identifier, à un stade précoce du développement préclinique et clinique des médicaments, les enzymes métabolisant ces médicaments ; étudier leur variabilité génétique, ainsi que celle des cibles pharmacologiques, et établir autant que possible les relations génotype-phénotype.

- Evaluer l'intérêt des tests génétiques non seulement lors d'études de pharmacocinétique et de tolérance, mais aussi au cours d'essais cliniques d'intervention conçus pour répondre à cette question.

- Constituer des banques d'ADN lors des grands essais cliniques d'intervention et analyser les points terminaux, et l'effet des traitements, en fonction du polymorphisme des gènes potentiellement impliqués dans l'effet thérapeutique et dans les contre-régulations physiologiques.

Améliorer les dispositions logistiques et réglementaires pour les études de génétique humaine : développer les centres de ressources biologiques pour la conservation des banques d'ADN, en prenant en considération les souhaits des industriels pour la gestion de leurs propres collections ; reconsidérer, en concertation avec les CCPPRB et l'Agence française de sécurité sanitaire des produits de santé (Afssaps) les procédures de mise en route des études génétiques et les règles de rédaction des consentements éclairés pour les prélèvements d'ADN afin de permettre une utilisation plus large de ces prélèvements, dans le respect des règles de bioéthique et de confidentialité.

\section{Participants}

P.Y. Arnoux (LEEM, Paris), E. Baumelou (CHRU, DRC, Lille), P.H. Beaune (INSERM U 490, Paris), J.P. Boissel (Pharmacologie Clinique EA 643, Lyon), F. Cambien (INSERM U 525, Paris), M. Cano-Petit (Aventis Pharma, Antony), J.F. Deleuze (Aventis, Evry), B. Diquet (Pitié-Salpétrière, Paris), J. Elion (INSERM U 458, Paris), L. Fluckiger (Afssaps, Saint-Denis), C. Funck-Brentano (CIC, Saint-Antoine, Paris), J.M. Job (LEEM, Paris), Ph. Lechat (Pharmacologie, Pitié-Salpétrière, Paris), L. Mignot (Hôpital Foch, Suresnes), S. Mouly (Hôpital Lariboisière, Paris), A. Parent de Curzon (Merck Sharp \& Dohme, Paris).

\section{Références}

1. Beaune P. Human cytochromes P450: applications in pharmacology. Thérapie 1993; 48 (6): 521-6

2. Meyer UA, Gut J. Genomics and the prediction of xenobiotic toxicity. Toxicology 2002; 181-182: 463-6

3. Boissel JP. Les espoirs de la pharmacogénétique : dialogue : la lettre des comités d'interface INSERM-Sociétés de spécialités médicales, Numéro 12, Octobre 2002 [online]. Available from URL: http://www.inserm.fr [Accessed 2003 Jul 14]

4. The European Agency for the Evaluation of Medicinal Products (EMEA). Evaluation of Medicines for Human Use: report to the CPMP (Committee on Proprietary Medicinal Products) on the use of pharmacogenetics in the drug 
development process, 5 Jun 2002 [online]. Available from URL: http://www.emea.eu.int/ pdfs/human/regaffair/148300en.pdf [Accessed $2003 \mathrm{Jul}$ 14]

5. Mancama D, Kerwin RW. Role of pharmacogenomics in individualising treatment with SSRIs. CNS Drugs 2003; 17 (3): 143-51

6. Nagasubramanian R, Innocenti F, Ratain MJ. Pharmacogenetics in cancer treatment. Annu Rev Med 2003; 54: 437-52

7. Humma LM, Terra SG. Pharmacogenetics and cardiovascular disease: impact on drug response and applications to disease management. Am J Health Syst Pharm 2002; 59 (13): 1241-52

8. Iqbal O. Pharmacogenomics in anticoagulant drug development. Pharmacogenomics 2002; 3 (6): 823-8

9. Marre M, Jeunemaitre X, Gallois Y, et al. Contribution of genetic polymorphism in the renin-angiotensin system to the development of renal complications in insulin-dependent diabetes: Génétique de la Néphropathie Diabétique (GENEDIAB) study group. J Clin Invest 1997; 99 (7): 1585-95

10. Parving HH, Jacobsen P, Tarnow L, et al. Effect of deletion polymorphism of angiotensin converting enzyme gene on progression of diabetic nephropathy during inhibition of angiotensin converting enzyme: observational follow up study. BMJ 1996; 313 (7057): 591-4

11. Loi n ${ }^{\circ} 88-1138$ du 20 décembre 1988. Available from URL: http://www. legifrance.gouv.fr

12. Brazell C, Freeman A, Mosteller M. Maximizing the value of medicines by including pharmacogenetic research in drug development and surveillance. Br J Clin Pharmacol 2002; 53 (3): 224-31

13. Katz DA. From bench to bedside: a diagnostics framework for pharmacogenetics research. Mol Genet Metab 2002; 77 (1-2): 57-60

14. Roses AD. Genome-based pharmacogenetics and the pharmaceutical industry. Nat Rev Drug Discov 2002; 1 (7): 541-9

15. March R, Cheeseman K, Doherty M. Pharmacogenetics: legal, ethical and regulatory considerations. Pharmacogenomics 2001; 2 (4): 317-27

Correspondance et offprints : François Alhenc-Gelas, INSERM U 367, 17 rue du Fer à Moulin, 75005 Paris, France.

E-mail : fagu367@ifm.inserm.fr 